\title{
La stérilisation par les moyens physiques et chimiques et son utilisation dans la lutte
} contre les insectes vecteurs

\author{
par J. MOUCHE'T
}

Directeur de Recherches, Services Scientifiques Centraux, O.R.S.T.O.M., F-93 - Bondy

\section{Résumé}

Ce papier est une revue des techniques utilisées dans la stérilisation physique ou chimique des insectes. L'action physiologique des rayons et chimiostérilisants, leur incidence sur l'activité, la longévité et la compétitivité sont traitées dans la deuxième partie qui envisage aussi leur toxicité pour les mammifères et les éventuelles résistances.

La dernière partie est un bilan des expériences de terrain réalisées à l'aide de ces techniques. Il y apparaît que des résultats intéressants ont été obtenus, notamment en ce qui concerne Cochliomyia hominivorax et la mouche domestique. Mais les perspectives à court terme sont moins encourageantes en ce qui concerne les moustiques. De nombreuses recherches d'écologie, d'éthologie, de génétique des populations sont encore indispensables pour rendre opérationnelle la lutte génétique contre les moustiques. En outre, se pose le problème de la production de masse d'individus compétitifs.

\section{Summary}

This paper is a review of the physical and chemical technics used in the male sterile control methods.

Physiological activity of radiations and chemosterilants is discussed in the second part dealing also with their incidence on insect activity, longevity and competitivness. Mammal toxicity and insect 
resistance to chemosterilants are briefly reported. The last part relate field experiments. Some interesting results have been obtained with the screw-worm fly and house fly. But mosquito genetical control to become operational needs much more research on ecology, behaviour and population genetics. The problem of mass production of competitive males is far from being solved.

\section{Introduction.}

Les succès obtenus dans l'éradication du «screw-worm» (Cochliomyia hominivorax Coquerel) aux Etats-Unis (Lindquist, 1961) ont considérablement stimulé les recherches sur l'utilisation de la stérilisation sexuelle pour la lutte contre les insectes. Knipling (1962) a montré tous les avantages théoriques de cette méthode pour une éradication plus rapide des espèces qu'avec les insecticides. Les généticiens ont produit des mâles hybrides stériles ou utilisé les incompatibilités cytoplasmiques pour la lutte génétique, alors que physiciens et chimistes ont proposé aux entomologistes une large gamme d'agents susceptibles d'induire la stérilité. C'est de cet aspect que nous allons parler.

Si des succès incontestables ont été enregistrés, il ne semble pas que l'on ait rencontré jusqu'ici des circonstances aussi favorables que lors des premiers travaux d'éradication du «screw-worm ». Mais un nombre considérable de recherches, tant au laboratoire que sur le terrain, sont en cours. Ces dernières ont mis clairement en évidence les insuffisances de nos connaissances de l'écologie, du comportement, de la génétique des populations.

Après un exposé sur les agents stérilisants et leur action physiologique, nous relaterons les résultats des travaux de terrain.

\section{Les agents stérilisants.}

\subsection{Agents physiques.}

L'effet stérilisant des rayons X est connu depuis 1916, sur le Coléoptère Lasioderma serricorne. Mais ce sont surtout les rayons gamma qui ont été utilisés, avec un succès particulièrement spectaculaire, lors de l'éradication de Cochliomyia hominivorax en Floride. La source radioactive la plus généralement employée est le cobalt 60 .

En fait, on a pu stériliser presque tous les arthropodes sur lesquels cette technique a été utilisée: Glossines, Mouches, Moustiques, Réduvides, etc. Mais la marge de sécurité entre la dose stérilisante et la dose nocive est souvent faible.

Par ailleurs, le nombre des mutations des Drosophiles nourries sur milieu irradié est augmenté (Swaminatham et al., 1963), alors qu'au contraire la fécondité de Phaenicia cuprina n'est pas altérée par les repas de viande irradiée (Morgan et Labrecque, 1964).

Les rayons bêta, peu pénétrants, sont inutilisables, mais l'ingestion d'isotopes $\mathrm{P}^{32}$, 
$\mathrm{Sr}^{89}$ et $\mathrm{S}^{35}$ émettant ces radiations a amené la stérilité des femelles d'Habrobracon (Grosch et al., 1956).

Les rayons ultraviolets provoquent une certaine stérilité chez les Aedes aegypti (Riordan, 1964 et 1966), et cette action pourrait être utilisée pour une autostérilisation dans des pièges à ultraviolets.

Enfin, le froid chez Argyroploce leucotrea (Myburg, 1965) et la chaleur chez Locusta migratoria (Yakimovich et Lusternak, 1965) sont susceptibles de provoquer une réduction de l'oogénèse et de la spermatogénèse.

\subsection{Agents Chimiques.}

Une revue complète des chimiostérilisants et de leurs propriétés physiologiques a été publiée en 1966 par Borkovec, et nous adoptons la classification de cet auteur.

\subsubsection{Agents alkylants.}

Ce sont des produits susceptibles d'introduire une chaîne hydrocarbonée dans une molécule organique. Sont seuls valables ceux qui produisent cet effet dans des conditions compatibles avec la vie.

Les Aziridines sont caractérisées par la présence de groupements éthyléneimines (= aziridines) dans leur formule, mais leur activité n'est pas proportionnelle au nombre de ces groupements.<smiles></smiles>

Les produits les plus actifs et les plus utilisés sont l'Apholate, la Tretamine, le Tepa, le Metepa et le Thiotepa. Mais, plus récemment, de nombreux autres composés ont été testés, dont l'Uredepa et Olim 53139 (Mulla, 1968). Une liste de ces produits avec leur formule est adjointe dans les annexes 1 et 2 .

Les Moutardes à l'azote sont jusqu'ici d'importance mineure.

\subsubsection{Les Antimétabolites.}

Ils interfèrent dans la synthèse des acides nucléiques et sont surtout actifs sur les femelles lorsqu'ils sont administrés au stade adulte.

Les analogues de la purine et de la pyrimidine entreraient en compétition avec les nucléotides et empêcheraient leur incorporation dans les acides nucléiques. Ils agiraient surtout au moment de la replication des chromosomes (Crystal, 1963). Le 5-Fluorouracyl n'empêche pas la ponte des mouches, mais les œufs sont stériles. Chez Aedes aegypti, il inhibe la digestion du sang et, de ce fait, bloque l'ovogénèse (Akov, 1965). Son action est temporaire. L'acide 5-fluoro-orotique a une action définitive et inhibe la ponte. 
Les antifoliques, Aminopterine et Methotrexate, ont une action temporaire sur l'ovogénèse ; cet effet peut être annulé, dans certains cas, par l'administration d'acide folique.

L'antagoniste de l'acide glutamique, le 6-diazo-5-oxonorleucine, provoque par voie topique la stérilisation de Cochliomyia hominivorax (Crystal, 1963).

\subsubsection{Les dérivés de la s-triazine et de la triamide phosphorique.}

Les dérivés de la s-triazine sont bien connus comme herbicides, mais beaucoup d'entre eux possèdent une certaine activité stérilisante.

Un certain nombre de ces produits sont alkylants et ont été cités en 2.2.1. Parmi les non-alkylants, le Hemel, homologue de la Tretamine, peu toxique, est plus actif sur les mâles que sur les femelles. L'Ipazine diminue le taux d'éclosion et de pupaison des mouches (Borkovec, 1967). La stérilité des mouches mâles par voie orale est plus difficile à obtenir (Fye et al., 1966).

Parmi les dérivés de la triamide phosphorique, le Hempa, homologue non alkylant du Tepa, s'est révélé très prometteur.

\subsubsection{Composés synthétiques divers.}

D'autres groupes de composés présentent des propriétés stérilisantes :

- les dérivés du Triphénylétain, très toxiques pour l'insecte (Kenaga, 1965) ;

- le 4-Imidazoline-2-one, inhibiteur de croissance (Schaefer et Tieman, 1967);

- la Réserpine, également très toxique pour les mâles de mouches (Wicht et Hays, 1967) ;

- les composés organiques du bore (Alkoxyboranes) sont actifs sur C. hominivorax (Settepani et al., 1969).

Quelques dérivés cycliques de l'urée ou de la thio-urée ont des propriétés stérilisantes sur les femelles; seule la 1-méthyl-1-nitroso-3-phénylurée agit sur les deux sexes de la mouche (Fujita et al., 1969), mais est très toxique.

\subsubsection{Les antihormones et Phéromones.}

Le m-xylohydroquinone, utilisé dans les pilules contraceptives, provoque une bonne stérilité, mais quelquefois temporaire, des mâles de mouches, sans affecter leur longévité (Ascher et Advat, 1966 et 1967).

La Phéromone (Ecthormone) du tégument de la reine d'abeille inhibe le développement ovarien des ouvrières. Le produit synthétique 9-ozodec-trans-2-enoil-acid injecté à Musca domestica inhibe temporairement la croissance ovarienne (Nayar, 1963).

\subsubsection{Les antibiotiques.}

La cycloheximide, utilisée comme systémique, inhibe la croissance des cellules nourricières de l'ovaire des acariens du genre Tetranychus (Harries, 1963). 
L'Hygromycine B et la Vancomycine sont inhibiteurs de reproduction du puceron Myzus persicae, ainsi qu'à un degré moindre l'Antimycine, la Fungichromine, la Caudicidine, la Griseoviridine, la Brévicidine et la Streptomycine (Harries et Wiles, 1966). L'Auréomycine inhibe la ponte de Bracon hebetor (Grosch, 1963). Leur mode d'activité intéresserait la synthèse des protéines ou, dans le cas des Aphides, les symbiotes (Harries et Wiles, 1966).

\subsubsection{Les insecticides.}

Les ovicides acaricides comme le Tetradifon, le Chlorbenside, le Chlorfenson, le Kelthane, réduisent la production d'œufs (Ascher, 1964).

\section{Activité biologique des agents stérilisants.}

\subsection{PRincipes générauX.}

En laboratoire, de très nombreuses espèces d'Arthropodes et même de Vertébrés ont pu être stérilisées par voie physique ou chimique, et Borkovec (1966) en fournit une liste exhaustive. Parmi les espèces d'intérêt médical, ce sont tout d'abord Musca domestica et Cochliomyia hominivorax qui ont fait l'objet du plus grand nombre de travaux. Mais on y relève également des moustiques, Aedes aegypti, Culex pipiens fatigans, Anopheles quadrimaculatus, Anopheles pharoensis, Anopheles labranchiae, les glossines, les stomoxes (Stomoxys calcitrans), les blattes (Blatella germanica), les Réduvides (Rhodnius prolixus), les tiques (Boophilus, Ornithodoros tholozani), etc.

Les agents stérilisants peuvent agir de trois façons :

- empêcher la production d'œufs et de sperme ;

— provoquer la mort des œufs et du sperme déjà produits ;

- induire des mutations létales dominantes dans les gamètes qui arrivent à maturation et participent à la formation du zygote, mais celui-ci ne donne pas de descendance (Proverbs, 1969). C'est cette dernière voie qui offre les meilleures perspectives pour la lutte contre les insectes.

La toxicité pour l'insecte et le pouvoir stérilisant d'un produit varient suivant les espèces, le mode d'administration (Gouck et al., 1963), la température (Crystal, 1967) et l'état physiologique de l'insecte (Crystal, 1966), et même l'heure de la journée (Crystal, 1969).

Les chimiostérilisants peuvent être utilisés par ingestion avec des appâts ou par contact (application topique, poudrage, aérosol, contact tarsal, bain, passage à travers de la mousse de polystyrène traitée) sur les stades adultes ou pré-imaginaux des insectes (Dame et al., $1964 a$; Dame et Schmidt, 1964 ; Crystal, 1964 et 1965 ; Fye et al., 1968). 


\subsection{Action physiologiQue.}

\subsubsection{Effets généraux.}

Les rayons gamma briseurs de chromosomes et les agents alkylants attaquant les radicaux phosphorés de l'A.D.N. ont un effet mutagénique et cytotoxique (Fahmy et Fahmy, 1964).

Ces deux types d'agents stérilisants appliqués précocement inhibent l'oogénèse et la spermatogénèse alors qu'un traitement plus tardif affecte peu les cellules des gonades, permet la formation des œufs et du sperme, mais induit les mutations léthales dominantes (Lachance et Leverich, 1962 ; Lachance et al., 1967 ; Riemann, 1967). Etant donné que c'est précisément ce dernier effet qui est recherché, il conviendra donc d'appliquer les traitements le plus tardivement possible au cours du cycle de l'insecte. Il faut noter que dans quelques cas la mutation létale induite est récessive (Palmquist et Lachance, 1966).

Laven (1969) propose l'utilisation des rayons X pour obtenir des translocations se traduisant par une semi-stérilité qui devrait amener l'éradication en quelques générations de C. pipiens. Curtis (1968) arrive aux mêmes conclusions avec Glossina austeni.

Le zygote, dont un des éléments est porteur de mutation, meurt généralement au cours du développement de l'embryon avant la formation du blastoderme chez $C$. hominivorax, mais quelquefois le développement peut se poursuivre jusqu'à la nymphose. La mort est due à des accidents mitotiques (Lachance et Riemann, 1964 ; Lachance et al., 1968).

D’une façon générale, les dommages causés aux cellules somatiques sont de même nature que ceux subis par les lignées germinales. Leur matériel génétique peut être atteint, même lorsqu'il n'y a pas de lésions cellulaires (Proverbs, 1969) et des altérations des chromosomes somatiques ont été observées (Rai, 1964 ; Lachance et al., 1968).

Cette action sur les cellules somatiques, beaucoup plus marquée avec les rayons gamma qu'avec les chimiostérilisants, peut se traduire par une diminution de la longévité (Flint, 1965) ou une baisse d'activité (Baumhover, 1965), notamment dans le domaine de l'agressivité sexuelle. Ces propositions sont toutefois contestées globalement ou partiellement par un certain nombre d'auteurs (Féron, comm. pers.).

Rayons et chimiostérilisants peuvent perturber la digestion sanguine par action sur les protéases (Akov, 1966).

\subsubsection{Effets sur les mâles.}

D'une façon générale, les dominants léthaux sont induits plus facilement aux stades précoces de la spermatogénèse que chez les spermatozoïdes (Savagham, 1963 ; Proverbs, 1969). Chez Culex fatigans, la marge entre les doses stérilisantes et toxiques est très faible pour les composés utilisables (Mulla, 1964). Chez Aedes aegypti, le traitement des larves à l'Apholate détériore le matériel génétique alors que les testicules 
sont normaux mais moins productifs (Rai, 1965). On observe également une déplétion du sperme (Sharma et Rai, 1967).

Le traitement des mâles d'Aedes aegypti au Thiotepa n'affecte pas la mobilité du sperme et provoque une stérilité complète mais partiellement réversible (Bertram, 1963). Les mêmes observations sont valables pour les mouches traitées au Thietepa (Sacca et al., 1964 ; Chang, 1965).

La question de la compétitivité des mâles est une des pierres fachoppement de la lutte génétique, et les résultats du laboratoire ne sont pas toujou Gérifiés sur le terrain. Elle dépend du type de stérilité, du comportement d'accouplement de l'espèce, de la longévité et de l'activité générale des mâles y compris de leur pouvoir de vol, leur aptitude à trouver les femelles dans la nature, et de leur agressivité sexuelle (Proverbs, 1969). Lorsque la femelle est polyandre, il faut que le sperme soit compétitif et mobile, ce qui n'est pas indispensable dans le cas de femelles monoandres. Chez la mouche, la réponse monoandre peut être déclenchée sans transfert de sperme. La fécondation même inefficace peut inhiber le transfert de sperme vers la spermathèque lors d'inséminations ultérieures.

Les expériences de laboratoire ne permettent de juger la compétitivité que pour l'accouplement proprement dit et, en partie, pour l'agressivité sexuelle. Aussi les résultats ne sont-ils pas toujours transposables au terrain.

Certains moustiques et $C$. hominivorax, stérilisés au Tepa et Metepa, ne seraient pas, en laboratoire, entièrement compétitifs (Dame et Schmidt, 1964, Crystal, 1964). La production insuffisante de sperme laisserait la spermathèque partiellement vide et susceptible d'être remplie ultérieurement de sperme fécond (Dame, Woodard et Ford, 1964).

Par contre, les mâles de mouches chimiostérilisés au Metepa ou à l'Apholate sont compétitifs (Murvosh et al., 1964 ; Labrecque et al., $1962 a$; Chang, 1965 ; Fye et Labrecque, 196 ). Certains mâles peuvent même transporter assez de chimiostérilisant dans leur sperme pour stériliser les femelles avec lesquelles ils s'accouplent (Borkovec, 1966).

Les mâles de Glossina pallidipes traités aux rayons gamma et de G. morsitans traités au Tepa seraient hypercompátitifs (Dean et Clements, 1969 ; Dame et Ford, 1966), de même que les mouches stérilisées à la xylohydroquinone (Ascher et Advat, 1967) et C. hominivorax traité avec Olim 53139 (Crystal, 1969).

Les mâles d'Aedes aegypti soumis au Thiotepa au stade nymphal sont très compétitifs, mais ne vivent que deux semaines (White, 1966).

Enfin, il faut signaler que des mâles d'An. pharoensis irradiés et d'An. quadrimaculatus traités à l'Apholate étaient très compétitifs avec des mâles normaux de la même souche, mais beaucoup moins avec des mâles d'une souche sauvage (Tantawy et al., 1967 ; Dame et al., 1964 b). C'est un résultat prévisible mais inquiétant. Le même phénomène a été vérifié chez $M$. domestica (Fye et Labrecque, 1966). 


\subsubsection{Effets sur les femelles.}

Le lâcher de femelles stériles n'est pas un des moyens utilisés en lutte génétique, mais, dans la mesure où il est difficile de les séparer des mâles, on peut être amené à les relâcher en même temps que ceux-ci.

D'autre part, en plaçant sur le dos des mouches femelles de petites quantités de stérilisant ( « booby traps »), on a tenté de stériliser les mâles venant s'accoupler (Morgan, 1967 ; Meiffert et al., 1967).

Enfin, dans les cas d'autostérilisation par appâts dans la nature, les femelles sont touchées au même titre que les mâles, et même davantage, dans la mesure où elles ingèrent plus d'appâts et donc plus de chimiostérilisants (Crystal, 1965 ; Sacca et al., 1964). Leur stérilisation contribue à la réduction de la population totale (Labrecque et al., 1962 b).

Il y a un nombre considérable de travaux sur l'action physiologique et cytologique des chimiostérilisants, qui sont résumés dans les revues de Borkovec (1966) et de Proverbs (1969) entre autres.

Généralement, la stérilité s'obtient plus facilement chez les femelles que chez les mâles, notamment par les antimétabolites.

L'oogénèse peut être inhibée par un traitement précoce des pupes. Les traitements plus tardifs induisent des mutations létales (Bertram, 1963 ; Crystal, 1963 ; Weidhaas, 1962 ; Dame et Ford, 1964 ; Crystal et Lachance, 1963).

Chez la mouche domestique et $C$. hominivorax, le Thiotepa et le Hempa, comme d'ailleurs la plupart des stérilisants, provoquent une dégénérescence des cellules folliculaires et nourricières dont les chromosomes sont lésés (Combiesco et al., $1967 a$ et $b$; Morgan, 1967 ; Lachance et Leverich, 1965). Ce type d'action se rencontre pratiquement chez tous les insectes. L'A.D.N. et l'A.R.N. montrent un déficit en acide adénylique et acide guanilique (Painter et Kilgore, 1967).

Chez les moustiques, l'action des aziridines, Tepa et Thiotepa est très rapide par exposition sur des surfaces traitées. Même lorsque les oocytes sont développés au stade V chez des femelles déjà inséminées, la stérilité peut être obtenue par détérioration des spermatozoïdes contenus dans la spermathèque; elle est moins marquée si les moustiques s'accouplent après le traitement (Weidhaas, 1962 ; Bertram, 1963 et $1964 a)$.

\subsubsection{Effets sur les stades pré-imaginaux.}

Des applications trop précoces de rayons gamma peuvent empêcher la pupaison par destruction de l'ecdysone tégumentaire (Kuzin et al., 1968).

Actuellement, le traitement nymphal est la méthode de choix pour la stérilisation en masse des moustiques d'élevage. Le Thiotepa à $1.000 \mathrm{ppm}$ a donné d'excellents résultats avec Aedes aegypti (White, 1966) et Culex fatigans (Weidhaas, comm. pers.).

Le traitement des larves peut amener la production d'adultes possédant des déformations, comme le cas est mentionné pour Blattella germanica traitée à l'Apholate (Burden et Smittle, 1963). 


\subsection{ACtion SUR LES PARASITES TRANSMIS.}

Du fait de leur excrétion rapide, les chimiostérilisants ne peuvent agir sur les parasites transmis que s'ils sont appliqués lorsque le parasite est déjà dans le vecteur ou immédiatement avant.

C'est sans doute dans le cas des trypanosomes que cette action est la plus intéressante à connaître, puisque, les glossines mâles étant vectrices, un lâcher de ceux-ci risquerait de créer une situation épidémiologique dangereuse. Les G. morsitans traitées au Tepa restent vectrices, mais à un degré moindre. La réduction est surtout marquée si le traitement a lieu lorsque la mouche est infectée, le Tepa ayant une action sur les formes cycliques des parasites (Dame et McKenzic, 1968).

Le taux de transmission de Plasmodium gallinaceum par Aedes aegypti est réduit par traitement du vecteur au Tepa et Thiotepa. Les périodes critiques pour le parasite se situent après leur ingestion, lors de l'exflagellation et à la $48^{\circ}$ heure lors de la méïose des oocytes (Altman, 1963 ; Bertram et al., 1964). La filaire Brugia patei dégénère chez les Aedes togoi traités au Thiotepa (Bertram, 1964 b). Mais dans les deux expérimentations, les vecteurs avaient été traités par des chimiostérilisants à doses létales.

\subsection{Métabolisme et excrétion.}

Le métabolisme du Metepa, Tepa et Thiotepa a été étudié chez les insectes et mammifères en utilisant des produits marqués au P $\mathrm{P}^{32}$ (Plapp et al., 1962 ; Parish et Arthur, 1965).

Le Metepa est rapidement excrété tel quel, ou après métabolisation, un des produits de dégradation le plus important étant l'acide phosphorique (Chamberlain et Hamilton, 1964). Chez le rat, l'excrétion est totale en 48 heures.

Chez l'insecte (B. germanica et St. calcitrans), le Thiotepa est transformé en Tepa puis excrété. Chez le rat, il y a en outre métabolisation en produits hydrolysables (Parish et Arthur, 1965).

La vitesse d'absorption et d'excrétion est variable suivant les espèces. C. hominivorax absorbe deux fois moins de Metepa que $S$. calcitrans et l'excrète deux fois plus vite. La stérilisation du premier nommé demandera donc des doses plus élevées que celle du second (Chamberlain et Hamilton, 1964).

L'excrétion du Metepa $\mathrm{P}^{32}$ par An. quadrimaculatus, Ae. aegypti et $M$. domestica est rapide, mais une certaine quantité des produits de dégradation est incorporée dans la synthèse de la matière vivante de l'insecte qui reste radioactif (Dame et Schmidt, 1964).

\subsection{ACTION SUR L'HOMME ET LES MAMMIFÈRES.}

L'ensemble de cette question a été révisé en 1968 par Hayes. La toxicité des chimiostérilisants varie grandement d'un produit à l'autre, même si les modifications de la formule sont mineures. Metepa et Apholate, en dose unique, ont la toxicité du 
D.D.T. et le Tepa celle de la Dieldrine, mais de faibles doses répétées peuvent provoquer la stérilité du rat et l'atrophie réversible du testicule (Gaines et Kimbrough, 1964). Le Hempa, non alkylant, a une toxicité 100 fois moindre que le Tepa pour une action stérilisante 50 fois moindre. Le Hemel est également moins toxique que la Tretamine (Chang, et al., 1964).

Le Thiotepa à doses répétées produit une stérilité du rat dont le sperme reste compétitif. Une action trop prolongée peut amener une destruction de l'épithélium séminal, le produit ayant un effet cumulatif. La Tretamine produit les mêmes effets (Jackson et al., 1959 ; Jackson et al., 1961 ; Boch et Jackson, 1957).

Les recherches dans la chimiothérapie des cancers ont fait ressortir pour les agents alkylants, un effet carcinogénique moindre que prévu. Thiotepa et Tretamine sont relativement bien tolérés avec, toutefois, de grandes variations de susceptibilité individuelle.

Du fait de leurs effets biologiques, les agents alkylants ne peuvent être utilisés ni en house spraying, ni dans le traitement des gîtes larvaires de moustiques (Barnes, 1964 ; Hayes, 1964).

\subsection{RÉSISTANCE.}

Par sélection larvaire, on a pu obtenir une souche d'Ae. aegypti 5 fois moins sensible à l'action stérilisante de l'Apholate (Hazard et al., 1964). Cette « résistance » amène également une moindre sensibilité au Tepa et au Metepa (Patterson et al., 1967). Klassen et Matsumura (1966) ont observé également une faible résistance au Tepa. La baisse de sensibilité à l'Hempa signalée par George et Brown (1967) était compensée par l'inbreeding et l'accumulation de gènes défavorables.

Sacca et Scirocchi, 1966, ont obtenu une résistance, au Metepa, de $M$. domestica, sur le plan de la stérilité, mais en même temps, la sensibilité à la toxicité augmentait. Abasa et Hansens (1969) ont obtenu une souche résistante à l'Apholate à partir d'une souche déjà résistante aux organophosphorés.

Enfin Ascher (1964) a signalé que le Tepa était peu actif sur une souche de mouches résistantes aux organophosphorés.

\section{Travaux de terrain.}

Le nombre des travaux même expérimentaux exécutés sur le terrain est relativement réduit, dans le domaine des insectes d'intérêt médical, comparativement aux travaux de laboratoire, et ils portent sur quelques espèces seulement.

\subsection{Cochliomyia hominivorax (Coquerel).}

Ce Diptère Calliphoridae a une répartition néo-tropicale jusqu'aux confins du Mexique. Il a envahi le Sud-Est des Etats-Unis vers 1933. Ses larves («screw-worm») provoquent des myiases extrêmement sérieuses du bétail. 
Les premières expériences de lutte biologique contre cet insecte se déroulèrent de 1951 à 1953 dans une île inhabitée de Floride (in Bushland, 1963), suivies de travaux plus importants à Curaçao en 1954 (Baumhover et al., 1955). Par des lâchers de mâles stérilisés aux rayons gamma, à raison de 400 individus par mille carré, on obtint son éradication de l'île en 6 mois.

Ces succès initiaux furent le prélude à une grande campagne dans le Sud-Est des Etats-Unis (Floride, Géorgie, Alabama). Des lâchers de 500 mâles stérilisés au mille carré amenèrent une rapide disparition de l'espèce en 1958 et 1959. L'usine qui avait été construite pour la production de masse de ces insectes fut même fermée (Weidhaas et al., 1960).

Dès 1960, réapparurent en Floride de petits foyers vite contrôlés par de nouveaux lâchers de mâles stérilisés.

Le succès de cette opération fut vraiment le catalyseur des recherches sur la lutte génétique. L'éradication de l'espèce dans son aire de répartition naturelle devint l'objectif de nouveaux travaux dans le Sud-Ouest des Etats-Unis et le Nord du Mexique dès 1962. Les essais expérimentaux de Veracruz, Mexique, en 1964 étaient prometteurs, mais pas conclusifs. Des lâchers de 400 mâles stérilisés par mille carré, diminuaient la densité sans faire disparaître l'espèce, peut-être par suite de réinvasions (Hightower et al., 1968). Les opérations prenaient néanmoins de l'ampleur, et en 1968, plus de 7 milliards de mâles stérilisés étaient relâchés sur le Sud-Ouest des Etats-Unis et le Nord du Mexique. Il se produisit néanmoins une des plus sérieuses «épidémies 》 de screw-worm enregistrées aux Etats-Unis (De Meillon, 1969).

Ce dernier résultat est un peu déconcertant. Il est incontestable que l'opération de Floride avait bénéficié de facteurs favorables du fait de l'isolement d'une population récemment introduite, et que la lutte dans l'aire naturelle de répartition d'un insecte est plus difficile. Il faudra néanmoins attendre confirmation et analyse des résultats du Sud-Ouest des Etats-Unis pour tirer des conclusions.

\subsection{Musca domestica L.}

Les expériences contre $M$. domestica ont ceci de particulier qu'elles ont été basées sur l'autostérilisation des populations autochtones par des appâts traités avec des chimiostérilisants. Il n'y a donc pas eu de problèmes résultant de l'introduction d'éléments étrangers dans une population naturelle.

Labrecque et al. $(1962 b)$, dans une île des Florida Keys, utilisèrent le Tepa à $0,5 \%$, sur des appâts sucrés disposés sur les champs d'épandage. La densité des mouches tendit vers 0 , et le pourcentage d'œufs fertiles fut de $1 \%$ seulement après 5 semaines. Des résultats analogues furent obtenus par Hansens et Granett (1963) et Gouck et al. (1963), à Pine Island en Floride.

Dans des élevages de poulets, donc sur des populations non isolées, Labrecque et al. (1963) et Labrecque et Meiffert (1966) obtinrent de très fortes baisses de densité avec le Metepa 0,5\%, et l'Apholate. Dans le dernier cas, les résultats étaient supérieurs à ceux de l'insecticide Trichlorfon. Mais on doit renouveler très souvent les appâts pour obtenir un fort pourcentage de stérilité des œufs. 
A Grand Turk Island, dans les Caraïbes, Labrecque et Keller (1965) réduisirent considérablement la population de l'île, en utilisant des appâts au Metepa $1 \%$. Mais l'éradication ne fut pas obtenue, car certains locaux comme les cuisines ne pouvaient être traités. Néanmoins, le contrôle fut meilleur qu'avec Trichlorfon (Meiffert et al., 1967).

En Italie, d'excellents résultats furent obtenus dans des champs d'épandage avec le Tepa et le Hempa. Le produit mélangé au sirop était pulvérisé sur les surfaces à traiter (Sacca et Stella, 1964 ; Sacca et al., 1966).

Il faut noter que tous ces résultats vont plus dans le sens du contrôle que de l'éradication, les populations se reconstituant rapidement à la cessation du traitement. D'ailleurs, des essais de lutte intégrée avec le Hempa et un insecticide ont donné d'excellents résultats en Floride.

\subsection{Lucilia sericata. Meigen.}

Dans une île de la côte Est de la Grande-Bretagne, où la population de Lucilia sericata était estimée à 2.000 mâles, des lâchers de mâles radiostérilisés en nombre supérieur à ceux de la population locale, ne donnèrent pas de résultats concluants. Il est possible que cet échec soit dû à une mauvaise corrélation entre les lieux des lâchers et les foyers naturels, ainsi qu'à des conditions climatiques défavorables (Donnelly, 1964).

\subsection{Haematobia irRitans L.}

D'après des expériences de laboratoire, les mâles de cette mouche radiostérilisés à $2.500 \mathrm{r}$ n'étaient pas complètement compétitifs. Des lâchers furent néanmoins exécutés en 1966 et 1967, dans une aire semi-isolée du Texas. Ils amenèrent une baisse des densités larvaires et imaginales non concluante. Les essais de lutte intégrée alliant l'utilisation, du Dichlorvos et les lâchers de mâles stériles se sont révélés meilleurs que le dernier procédé seul (Hoffman et Schmidt, 1968).

\subsection{Les Glossines.}

En laboratoire, l'utilisation des rayons gamma provoqua quelques déboires, diminuant la longévité des mâles traités (Potts, 1964). Toutefois, lorsque le traitement est effectué après le $16^{\circ}$ jour de la pupaison, la plupart des effets défavorables disparaissent (Dean et al., 1968). Itard (1968) stérilisa G. tachinoides sans affecter la vigueur des mâles très compétitifs tout comme Dean et Clements (1968) avec G. pallidipes, mais dans ce dernier cas, la stérilisation fut subtotale. Toutefois, dans les essais en cage, où mâles normaux et traités étaient mélangés, le taux de reproduction fut inférieur aux prévisions.

L'Apholate et le Tepa, administrés aux nymphes, diminuent la longévité de $G$. morsitans (Chadwick, 1964). Sur la même espèce, Dame et Ford (1966) provoquèrent une stérilité totale en traitant les mâles au Tepa par contact tarsal. La compétitivité était bonne, et le sperme normal. 
Challier (1966) avait fait remarquer que les accouplements multiples pourraient faire obstacle à des campagnes de lutte.

D'autres expériences sont projetées en République Centrafricaine.

Les méthodes d'élevage des tsétsés, bien qu'améliorées, restent très délicates, et le faible taux de reproduction de ces insectes rend leur production de masse très difficile.

Un projet pilote dans une île de Rhodésie avait été prévu en 1966 pour une lutte intégrée contre G. morsitans. On devait stériliser des pupes prélevées dans la nature et relâcher les mâles après un traitement insecticide pour abaisser la population (Phelps, 1967). Nous n'avons pas connaissance des suites de ce projet.

\subsection{CUlex PIPIENS Fatigans Wiedemann.}

Après les expériences préliminaires au laboratoire (Ramakrishnan et al., 1962), un essai de lutte contre $C$. fatigans fut exécuté en Inde avec des mâles stérilisés aux rayons gamma. La population locale ne diminua pas, mais il y eut un accroissement de la proportion des œufs non embryonnés. L'expérience fut interrompue par suite de l'opposition des habitants. Une des causes d'échec serait le nombre insuffisant des mâles relâchés (Krishnamurthy et al., 1962).

En Floride, $C$. fatigans a pu être éradiqué d'une petite île par des lâchers de mâles stérilisés à l'état nymphal par le Thiotepa (Weidhaas, comm. pers., 1969).

Des essais de terrain vont avoir lieu en Tanzanie avec des mâles stérilisés oralement à l'Apholate (A. Smith, comm. pers.), et un projet de grande envergure va démarrer en Inde autour de Delhi.

Knipling et al. (1968) ont suggéré d'utiliser des mâles autostérilisés dans des pièges à U.V. enduits de Tepa.

\subsection{Aedes AegYPTi L.}

Une seule expérience a été réalisée dans deux localités isolées de Floride (Morlan et al., 1962). Les lâchers de pupes radiostérilisées n'ont eu aucun effet sur la population d'une des 2 localités, alors que dans l'autre, ils ont provoqué une baisse de densité. L'échec fut attribué à une mauvaise répartition des nymphes stériles et à une fréquence insuffisante des lâchers.

\subsection{Anophèles Quadrimaculatus Say.}

Des lâchers de mâles stériles dans des îles de Floride pendant 11 mois n'ont pas amené de baisse de densité ni de stérilité chez les femelles sauvages. Cet échec serait dû à la différence de comportement entre les populations sauvages et celles d'élevage (Weidhaas et al., 1962). Ceci a été d'ailleurs vérifié expérimentalement par Dame et al. $(1964 b)$. 


\subsection{Autres insectes.}

Il semble que des résultats plus substantiels aient été obtenus dans le domaine agricole.

Dacus cucurbitae a été éradiqué d'une des îles Mariannes (Steiner et al., 1965).

Carpocapsa pomonella aux Etats-Unis a été contrôlé dans d'aussi bonnes conditions par les lâchers de mâles stériles que par les insecticides (Proverbs, 1969).

Dans le Sud-Ouest des Etats-Unis, les lâchers de mâles stérilisés au Tepa ont remplacé les insecticides pour la lutte contre la mouche des fruits mexicaine (Anastrepha ludens) (Proverbs, 1969). On a également préconisé l'autostérilisation des mâles attirés dans un piège en leur offrant une eau traitée avec un chimiostérilisant (Borkovec, 1966).

La lutte contre la mouche des fruits méditerranéenne (Ceratitis capitata) par des lâchers de mâles radiostérilisés, a enregistré des succès un peu partout, et en Amérique centrale on a pu arriver à une réduction sensible des dommages causés aux agrumes (in Proverbs, 1969).

\section{Conclusions.}

Il apparaît clairement que les résultats obtenus dans la lutte génétique contre les vecteurs ne sont pas encore suffisants surtout en ce qui concerne les moustiques. Les échecs enregistrés ne condamnent pas pour autant les méthodes génétiques, mais font apparaître la nécessité de recherches très approfondies.

Tout d'abord, il faut améliorer les techniques de stérilisation de façon à ce qu'elles ne lèsent en aucune façon les spécimens à relâcher.

Mais ce sont les connaissances précises sur l'écologie, notamment dans son aspect quantitatif, l'éthologie et plus particulièrement le comportement sexuel ainsi que la génétique des populations qui font le plus défaut.

Les méthodes d'élevage de masse des insectes doivent être envisagées sur le double aspect de la production de grandes quantités de spécimens et de l'obtention d'individus compétitifs avec les populations à combattre.

L'autostérilisation des populations naturelles est rarement possible, sauf dans le cas des mouches. Mais il n'est pas exclu que l'on puisse utiliser des spécimens pris sur le terrain pour des lâchers ultérieurs. Les méthodes de piégeage et les attractifs actuellement disponibles sont encore insuffisants, et doivent être améliorés.

De toute façon, les méthodes de lutte génétique différeront pour chaque espèce, et il serait illusoire d'y voir une panacée adaptable à tous les cas. Mais on peut raisonnablement espérer que l'éradication du \&screw-worm » ne restera pas un cas isolé, et que des techniques biologiques appropriées viendront s'inscrire dans les programmes de lutte intégrée contre les vecteurs. 
ANNEXE 1

\section{Agents stérilisants}

AZIRIDINES ET ANALOGUes.

Apholate : 2,2,4,4,6,6,-hexahydro-2,2,4,4,6,6-hexakis-(1-aziridinyl)-1,3,5,2,6-triazine-tri-phosphorine.

Tepa : tris-(-1-aziridinyl)-phosphine-oxyde (= Aphoxide).

Tretamine : 2,4,6-tris-(-1-aziridinyl)-s-triazine.

Metepa : tris-(2-methyl-1-aziridinyl)-phosphine-oxide.

Thistepa : tris-(1-aziridinyl)-phosphine-sulfide.

Uredepa : Ethyl-bis-(1-aziridinyl)-phosphinyl-carbamate.

Olim 53139: N-N'-hexamethylène-bis-(1-aziridinyl)-carboxamine.

DÉRIVÉS DE LA S-TRIAZINE.

Hemel : tris-2-4-6-dimethyl-amino-s-triazine (homologue de la Tretamine).

DÉRIVÉS DE LA TRLAMIDE PHOSPHORIQUE.

Hempa: Hexamethyl phosphorique triamide (non alkylant homologue de Tepa).

ANTimétabolites.

5-Fluoro-uracil.

Acide-5-fluoroorotique.

Aminoptérine.

Méthotrexate-(= Améthopterine).

\section{ANTIHORMONE.}

m.-xylohydroquinose. 
ANNEXE 2
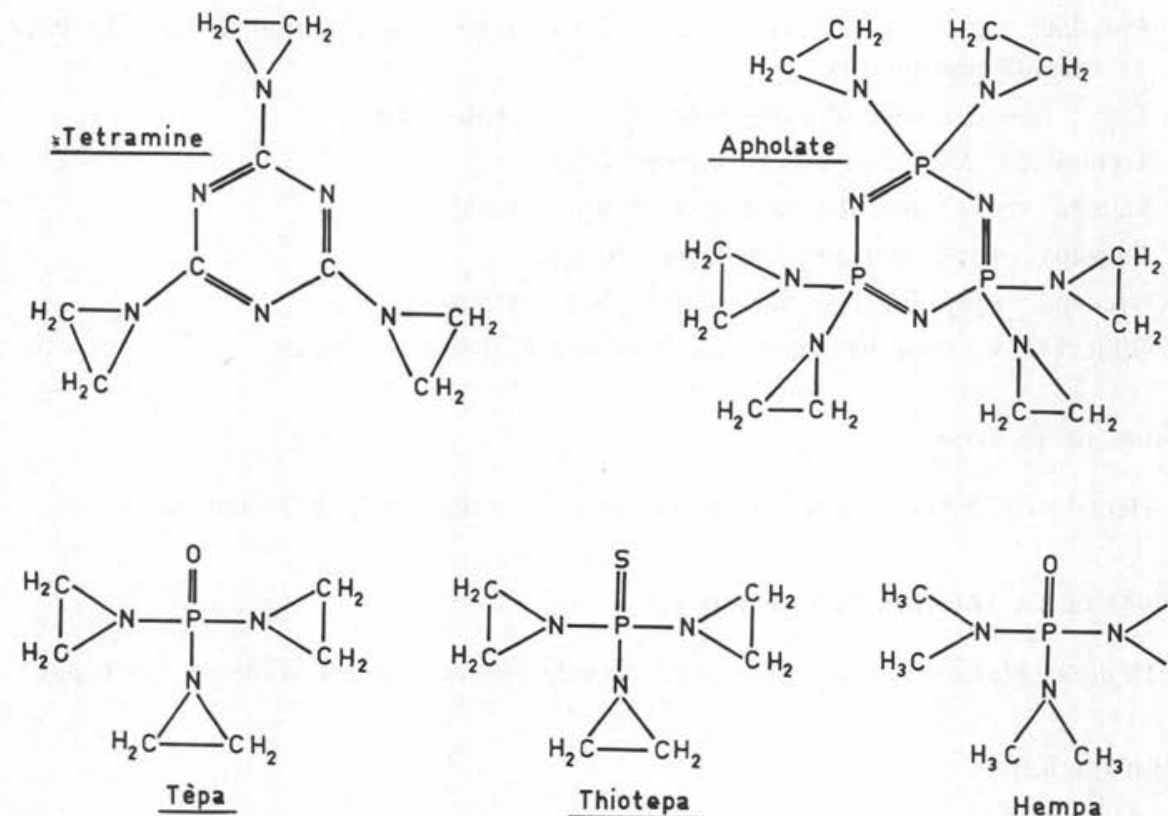<smiles>S=P(N1CC1)(N1CC1)N1CC1</smiles>

Thiotepa<smiles>CN(C)P(=O)(N(C)C)N(C)C</smiles>

Hempa<smiles>CC(F)=CNC(=O)NC(=O)c1ccc(N)nc1N</smiles>

5-Fluorouracil<smiles>Cc1cc(O)cc(C)c1O</smiles>

Xylohydroquinone
Methotrexate<smiles>O=C(O)C1CCCN1</smiles><smiles>CCC(=O)O</smiles> 


\section{Bibliographie}

Abasa (R. O.) et Hansen (E. J.), 1969. - An apholate resistant strain of house flies. I. Resistance to other chemosterilant and to insecticide. J. econ. Ent., 62 (2) : 334-38.

Akov (A. S.), 1966. - Retention of the blood meal in Aedes aegypti following sterilization by chemicals and irradiation. Ann. trop. Med. Parasit., 60 (4) : 482-94.

Altman (R. M.), 1963. - The effects of Tepa on Plasmodium gallinaceum in Aedes aegypti. Am. J. Hyg., 77 : 221-227.

Ascher (K. R.S.), 1964. - A review of chemosterilants and oviposition-inhibitors in insects. World. Rev. Pest. Control, 3 (1): 7-27.

— et Avdat (N.), 1967. - Sterilizing the male housefly with m. xylohydroquinone. Int. Pest. Control, 9 (2) : 8-13.

BARNeS (J. M.), 1964. - A symposium on chemosterilants in pest and vector control. III. Toxic hazards and the use of insect chemosterilants. Trans. R. Soc. trop. Med. Hyg., 58 : 327-34.

Baumhover (A. H.), 1965. - Sexual aggressiveness of male screw-worm flies measured by effect on female mortality. J. econ. Ent., 58 (3) : 544-48.

— et al., 1955. - Screw-worm control through release of sterilized flies. J. econ. Ent., 48 : 462-66.

Bertram (D. S.), 1963. - Observations on the chemosterilant effect of an alkylating agent, Thiotepa on wild caught Anopheles gambiae melas (Theo) in Gambia, West Africa and in the laboratory. Trans. R. Soc. trop. Med. Hyg., $57: 322-35$.

-, 1964. - A symposium on chemosterilants in pest and vector control. I. Entomological and parasitological aspects of vector chemosterilization. Trans. R. Soc. trop. Med. Hyg., 58 (4) : 296-317.

-, SRivastava (S. C.) et Msangi (A. S.), 1964. - Transmission of Plasmodium gallinaceum to chicks by Aedes aegypti (L.) sterilized by an alkylating agent, Thiotepa. J. Trop. Med. Hyg., 57 : 51-57.

Boch (M.) et JACKSON (H.), 1957. - The action of triethylene melamine on the fertility of the male rats. Brit. J. Pharmacol., 12 : 1-7.

Borkovec (A. B.), 1966. - Insect chemosterilants. Advances in Pest control Research, 7, 114 pages.

-, Labrecque (G. C.) et de Millo (A. B.), 1967. - S. Triazine herbicides as chemosterilants of house flies. J. econ. Ent., 60 (3) : 893-94.

Burden (G. S.) et Smittle (B. J.), 1963. - Chemosterilant studies with the German Cockroach. Florida Entomologist, 46 (3) : 229-233.

Bushland (R. C.), 1963. - Male sterilization for the control of insects. Advances in Pest control Research, 3 : 1-25.

CHAdwick (P. R.), 1964. - Effects of two chemosterilants on Glossina morsitans. Nature, Lond., 204 (4955): 299-300.

Challier (A.), 1966. - Possibilités d'application de la technique du mâle stérile à la lutte contre les glossines. Rapp. final $V I^{\circ}$ Conf. techn. OCCGE, Bobo-Dioulasso, 21-26 mars 1966: 329-30. 
Chamberlain (W. F.) et Hamilton (E. W.), 1964. - Absorption, excretion and metabolism of $\mathrm{P}^{32}$ labelled Metepa by screw-worm and stable flies. J. econ. Ent., 57 (6) : 800803.

Chang (S. C.), 1965. - Chemosterilization and mating behavior in male House Flies. J. econ. Ent., 58 (4) : 609-672.

-, Terry (P. H.) et Borkovec (A. B.), 1964. - Insect chemosterilants with low toxicity for mammals. Science, 144 (3614) : 57-58.

Combiesco (I.), Duport (M.) et Enesco (A.), $1967 a$. - Stérilisation de l'espèce Musca domestica $L$. par traitement avec divers produits chimiques. Note 1. Recherches de laboratoire. Arch. roum. Path. exp. Microbiol., 26 (1) : 205-214.

-, Enesco (A.) et Ciplea, 1967 b. - Etude de l'action des chimiostérilisants. Thiotepa et Apholate, sur le développement des ovaires de l'espèce Musca domestica L. Note II. Arch. roum. Path. exp. Microbiol., 26 (1) : 215-27.

CRYSTAL (M. M.), 1963. - The induction of sexual sterility in the screw-worm fly by antimetabolites and alkylating agents. J. econ. Ent., 56 (4) : 468-73.

-, 1964. - Sexual sterilization of screw-worm flies by the biological alkylating agents, Tretamine and Thiotepa. Exp. Parasit., 15 : 249-59.

-, 1965. - Sexual sterilization of Insects by aerosol administration of alkylating agents. J. econ. Ent., 58 (4) : 678-680.

-, 1966. - Sexual sterilization of screw-worm flies by a peroral chemosterilant : quantitative aspects and relation to pretreatment starvation. J. econ. Ent., 59 (3) : 580-85.

-, 1967. - Chemosterilant effect of Tretamine enhanced in screw-worm flies exposed to extraoptimal temperature. J. econ. Ent., 60 (3): 880-81.

-, 1969. - Changes in susceptibility of screw-worm files to the chemosterilant NN' tetramethylenebis (1 aziridinecarboxamide) with time of administration. J. econ. Ent., 62 (1) : 275-76.

- et Lachance (L. E.), 1963. - The modification of insects treated with alkylating agents. I. Inhibition of ovarian growth and egg production and hatchability. Biol. Bull., 125 (2) : 270-79.

DAME (D. A.) et Ford (H. R.), 1964. - Chemosterilization and its permanency in mosquitoes. Nature, Lond., 201 (4920) : 733-734.

CURTIS (C. F.), 1968. - Studies on the feasibility of using chromosome translocations for tsetse eradication. Abst. Rev. 8th. Intern. Cong. Trop. Med. Malaria, Téhéran $1968: 346$.

DAME (D. A.) et Ford (H. R.), 1966. - Effect of the chemosterilant Tepa on Glossina morsitans Westw. Bull. ent. Res., 56 (4) : 649-58.

— et MacKenzIE (P. K. I.), 1968. - Transmission of Trypanosoma congolense by chemosterilized male G. morsitans. Ann. trop. Med. Parasit., 62 (3) : 372-74.

- et Schmidt (C. H.), 1964. - Uptake of Metepa and its effect on two species of mosquitoes (Anopheles quadrimaculatus, Aedes aegypti, and house flies Musca domestica). J. Econ. Ent., 57 (1) : 77-81.

-, Woodard (D. B.) et Ford (H. R.), $1964 a$. - Chemosterilization of Aedes aegypti by larval treatments. Mosq. News, $24: 1-6$. 
-, - , - et Weidhas (D. E.), 1964 b. - Field behaviour of sexually sterile Anopheles quadrimaculatus males. Mosq. News, 24 (1): 6-14.

Dean (G. J. W.) et Clements (S. A.), 1969. - Effect of gamma radiation on Glossina pallidipes Aust. Bull. ent. Res., 58 (4) : 755-762.

Dean (G. J.), Wilson (F.) et Wortham (S.), 1968. - Some factors affecting eclosion of Glossina morsitans from pupae. Bull. Ent. Res., 58 (2) : 367-77.

Donnelly (J.), 1964. - Possible causes of failure in a field test of the \&sterile males » method of control. Proc. 12th. Int. Cong. Ent. London 1964 : 253-54.

FAHMY (O. G.) et FaHMY (M. J.), 1964. - A symposium on chemosterilants in pest and vector control. II. The chemistry and genetics of the alkylating chemosterilants. Trans. R. Soc. Trop. Med. Hyg., 58 (4) : 318-326.

FLINT, 1965. - The effects of gamma radiation on the fertility and longevity of Hippelates pusio. J. econ. Ent., 58 (3) : 555-58.

Fujita (T.), Tsuji (H.), Deurat (H.) et Nakajima (M.), 1969. - Insect sterilization activity of the 1. Methyl 1 nitroso-3-phenylurea. Agric. biol. chem. Tokyo, 33 (5): 785-89.

Fye (R. L.) et Labrecque (G.C.), 1966. - Sexual acceptability of laboratory strains of male house flies in competition with wild strains. J. econ. Ent., 59 (3) : 538-40.

- , - et GoucK (H. K.), 1966. - Screening tests of chemicals for sterilisation of adult house flies. J. econ. Ent., 59 (2) : 485-87.

-, 一, Morgan (P. B.) et Bowman (M. C.), 1968. - Development of an autosterilization technique for the house fly. J. econ. Ent., 61 (6): 1578-81.

Gaines (T. B.) et Kimbrough (R. D.), 1964. - Toxicity of metepa to rats with notes on two other chemosterilants. Bull. Org. Mond. Santé, 31 (5): 737-45.

George (J. A.) et Brown (A. W. A.), 1967. - Effect of the chemosterilant Hempa on the yellow fever mosquito and its liability to induce resistance. J. econ. Ent., 60 (4): 974-78.

Gomez-NunEZ (J. C.), 1963-67. - Estudio sobre el posible control de Rhodnius prolixus por medio de radiacione ionizantes. Acta ci. venezol. suppl. 3 (Inst. Venezol. Invest. ci. Arch. cuatrienal 1963-67): 98-105.

Grosch (D. S.), 1963. - Insect fecundity and fertility; chemically induced decrease. Science, 140 (3582) : 732-33.

Gouck (H. K.), Meifert (D. W.) et Gahan (J. B.), 1963. - A field experiment with Apholate as a chemosterilant for the control of house flies. J. econ. Ent., 56 (4) : 445-46.

Grosch (D. S.), Sullivan (R. L.) et Lachance (L. E), 1956. - The comparative effectiveness of four beta emitting isotopes fed to Habrobracon females on production and hatchability of eggs. Radiat. Res., 5 : 281-89.

Hansens (E. J.) et Granett (P.), 1965. - Effects of Apholate on a restricted population of house flies. J. econ. Ent., 58 (1) : 157-58.

Harries (F. H.), 1963. - Effects of some antibiotics and other compounds on fertility and mortality of orchard mites. J. econ. Ent., 56 (4) : 438-41.

- et Wiles (W. G.), 1966. - Tests of some antibiotics and other chemosterilants on the green peach aphid. J. econ. Ent., 59 : 694-96. 
Hayes (W. J.), 1964. - The toxicology of chemosterilants. Bull. Org. Mond. Santé, 31 : 721-36.

-, 1968. - Toxicological aspects of chemosterilants. Principle of Insect Chemosterilisation, 315-47 (Labrecque et Smith Eds, Appleton Century Crofts, N. Y. 1968).

Hazard (E. I.), Lofgren (C.S), Woodard (D. B.), Ford (H. R.) et Glancey (B. M.), 1964. - Resistance to chemical sterilant, Apholate, in Aedes aegypti. Science, 145 (3631): 500-501.

Hightower (B. G.), Alley (D. A.) et Edde (J. C.), 1968. - Observations on the effects of releasing sterile screw-worm flies in Northern Vera Cruz, Mexico. Folia Ent. Mex., $17: 3-16$.

Hoffman (R. A.) et SCHMidt (C. D), 1968. - The effects of a field release of sterile male horn flies on a semi-isolated population. Folia ent. mex., 18-19 : 77-78.

ITARD (J.), 1968. - Stérilisation des mâles de Glossina tachinoides par irradiation aux rayons $\gamma$. Rev. Elev. Med. Vet. Pays Trop., 21 (4) : 479-91.

JaCKson (H.), Fox (B. W. et Craig (A. W), 1959. - Alkylating agents and fertility. Brit. J. Pharmacol., 14 : 149-157.

- , - - , 1961. - Antifertility substances and their assessment in the male rodent. $J$. Reproduct. Fert., 2 : 447-65.

Kenaga (E. E.), 1965. - Triphenyl Tin compounds as insect reproduction inhibitors. J. econ. Ent., 58 (1) : 4-9.

Klassen (W.) et Matsumura, 1966. - Resistance to a chemosterilant, Metepa in Aedes aegypti mosquitoes. Nature, 209 : 1155-56.

KNIPLING (E. F.), 1962. - Potentialities and progress in the development of chemosterilants for insect control. J. econ. Ent., 55 (5) : 782-86.

- et al., 1968. - Genetic control of insects of public health importance. Bull. Org. Mond. Santé, 38 (3) : 421-38.

Krishnamurthy (B. S.), RaY (S. N.) et Joshi (G.C.), 1962. - Summary and conclusions Indian Journ. of Malar., 16 (4) : 365-373.

KuZin (A. M.), Kolomistseva (I. K.) et Yusifov (N. I.), 1968. - Effect of ecdysone on the puparium formation in irradiated Ephestia kuhniella Z. Nature, $217: 743-44$.

Labrecque (G. C.) et Keller (J. C), 1965. - Advances in insect population control by the sterile-male technique. Intern. Atom. Energy Agency Tech. Rept. Ser., 44, 78 p.

- et Meifert (D. W.), 1966. - Control of house flies in poultry houses with chemoster1lants. J. med. Ent., 3 (3-4) : 323-26.

- , - et SмIтH (C. N.), $1962 a$. - Mating competitiveness of chemosterilized and normal male house flies. Science, 136 (3514) : 388-89.

- , - , $1962 \mathrm{~b}$. - Field experiment in the control of house flies with chemosterilant bait. Jour. econ. Ent., 55 (4) : 449.

-, - et FYE (R. L.), 1963. - A field study on the control of house flies with chemosterilant technique. J. econ. Ent., 56 (2) : 150-152.

LACHANCE (L. E.) et Leverich (A. P.), 1962. - Radiosensitivity of developping reproductive cell in female Cochliomyia hominivorax. Genetics, 47 : 721-735. 
- , - 1965. - Cytogenetic studies on the effect of an alkylating agent on insect nurse cell polytene chromosomes as related to ovarian growth and fecundity. Genetics, 52 : 453-54.

- et Riemann (J. G.), 1964. - Cytogenetic investigations on radiation and chemically induced dominant lethal mutations in oocytes and sperm of screw-worm fly. Mutation Res., 1 : 318-33.

-, Schmidt (C. H.) et Bushland (R. C.), 1967. - Radiation induced sterilization. Pest Control Biological Physical and selected chemical methods, 147-96 (Kilgore et Doutt Eds, Acad. Press. N. Y., 477 p.).

-, North (D. T.) et KLAssen (W.), 1968. - Cytogenetic and cellular basis of chemically induced sterility in insects. Principles of Insects chemosterilization, 99-157 (Labrecque et Smith Eds, New-York).

LAVEN (H.), 1969. - Eradicating mosquitoes using translocations. Nature, Lond., 221 (5184) : 958-59.

LindQuist (A. W.), 1961. - New ways to control insects. Pest Control, 29 : 9-20.

Meifert (D. W.), Morgan (P. B.) et Labrecque (G. C.), 1967. - Infertility induced in male house flies by sterilant bearing females. J. econ. Ent., 60 (5) : 1336-67.

-, Labrecque (G. C.), Smith (C. N.) et Morgan (P. B.), 1967. - Control of house flies on some West Indies Islands with Metepa, Apholate and Trichlorfon baits. J. econ. Ent., 60 (2) : 480-85.

MeIllon (B. DE), 1969. - Conference on Anopheline biology and Malaria eradication (May 21-23, 1969). Mosquito Systematics News Letter, 1 (3): 35-40.

Morgan (P. B.), 1967. - Booby trapped female house flies as sterilant carriers. J. econ. Ent., 60 (2): 612-13.

- et Labrecque (G. C.), 1964. - Studies on the effect of meat exposed to gamma radiation or chemosterilants on the reproductive capacity of a blow fly Phaenicia cuprina (Wied.). Florida Ent., 47 (1) : 31-33.

Morlan (H. B.), Cray (E. M.) et Kilpatrick (J. W.), 1962. - Field tests with sexually sterile males for control of Aedes aegypti. Mosquito News, 22 (3) : 295-300.

Mulla (M.S.), 1964. - Chemosterilization of the mosquito Culex p. quinquefasciatus. Mosq. News, 24 (2) : 212-217.

-, 1968. - Chemosterilants for control of reproduction in the eye gnat (Hippelates collusor) and the mosquito Culex quinquefasciatus. Hilgardia, 39 (10): 297-324.

Murvosh (C. M.), Labrecque (G. C.) et Smith (C. N.), 1964. - Effect of three chemosterilants on house fly longevity and sterility. J. econ. Ent., 57 (1) : 89-93.

MYburg (A. C.), 1965. - Low temperature sterilization on false codling moth Argyroploce leucotrea Meyr. in export citrus. J. Ent. Soc. S. Africa, 28 : 277-85,

NAYAR (J. K.), 1963. — Effect of synthetic «Queen substance » (9 oxodec-trans-2-enoil acid) on ovary development of the house fly Musca domestica L. Nature, 197 (4870) : 923-24.

Painter (R. H.) et Kilgore (W. W.), 1967. - The effect of Apholate and Thiotepa on nucleid acid synthesis and nucleotide ratios in housefly eggs. J. Insect. Physiol., 13 (7) : 1105-1118. 
Palmouist (J.) et Lachance (L. E.), 1966. - Comparative mutagenicity of two chemosterilants Tepa and Hempa in sperm of Bracon hebetor. Science, 154 : 915-17.

PARISH (J. C.) et ARTHUR (B. W.), 1965. - Mammalian and insect metabolism of the chemosterilant Thiotepa. J. econ. Ent., 58 (5) : 976-79.

Patterson (R. S.), Lofgren (C. S.) et Boston (M. D.), 1967. - Resistance in Aedes aegypti to chemosterilants, effect of Apholate selection on resistance to Apholate, Tepa and Metepa. J. econ. Ent., 60 (6) : 1673-1675.

Phelps (R. J.), 1967. - The sterile male technique in relation to tsetse fly control. Proc. Trans. Rhodesia Sc. Assoc., 52 (1) : 29-32.

Plapp (F. W.), Bigley (W. S.), Chapman (G. A.) et Eddy (G. W.), 1962. - Metabolism of Methaphoxide in Mosquitoes, House flies, and Mice. J. econ. Ent., 55 (5) : 607613.

PotTs (W. H.), 1964. - The gamma irradiation of Glossina puparial stages and control. Proc. 12th Int. Cong. Ent. London 1964 : 254-55.

Proverbs (M. B.), 1969. - Induced sterilization and control of insects. Ann. Rev. Ent., $14: 81-102$.

RAI (K. S.), 1964. - Cytogenetic effects of chemosterilants in mosquitoes. I. Apholate induced aberrations in the somatic chromosomes of Aedes aegypti. Cytologia (Tokyo), 29 : 346-53.

-, 1965. - Cytogenetics of chemosterilant induced sterility in the mosquito Aedes aegypti L. Proc. 12th Int. Cong. Ent. London: 255-56.

Ramakrishnan (S. P.) et Krishnamurthy (B. S.) et RaY (S. N.), 1962. - Laboratory studies on the use of irradiated sterile males to reduce $C$. fatigans Wied. Populations. Indian Jour. Malar., 16 (4) : 357-64.

RIEMANN (J.G.), 1967. - A cytological study of radiation effects in testes of the screwworm fly Cochliomyia hominivorax. Ann. ent. Soc. Amer., 60 : 308-20.

RIORDAN (D. F.), 1964. - Effects of high intensity flash discharge as a source of radiant energy for sterilizing mosquito. Nature, 204 : 13-32.

-, 1966. - Effects of high intensity light flashes on mortality and reproduction of Aedes aegypti. Can. J. Zool., $44:$ 895-902.

SACCA (G.), 1961. - Esperienze con mosche domestiche sterilizate con raggi. Atti Acad. naz. ital. Ent., 8 : 91-98.

- et ScIrocchi, 1966. - An attempt to select a strain of Musca domestica resistant to Metepa. WHO/Vector Control: 66-192.

-, -, de Meo (G. M.) et Mastrilli (M.), 1966. - Una prova di campo con il chemosterilante Hempa contro M. domestica L. Atti. Soc. pelor. Sci. fis. mat. nat. Messina, $12(1-2): 457$.

- et Stella (E.), 1964. - Una prova di campo per il controllo di Musca domestica L. mediante esche liquide a base del chemosterilante Tepa. Riv. Parassit., 25 : 279.

-, - et Megrone (R.), 1964. - Ricerche di laboratorio sul l'efficacia sterilizante del Tepa (Afoxide) e dell' Afolate in Musca domestica. Riv. Parassit., 25 : 207-16. 
Savagham (R.), 1963. - Cell stages and differential sensitivity to irradiation in males of Drosophila melanogaster. Repair for Genetic Radiation. Radiation Damage and Differential Radiosensitivity in germ cells: 343-57.

Schaefer (E. H.) et Tieman (E. H.), 1967. - 4-Imidazolin-2-one : an insect growth inhibitor and chemosterilant. J. econ. Ent., 60 (2) : 542-46.

Settepani (J. A.), Crystal (M. M.) et Borkovec (A. B.), 1969. - Boron chemosterilants against screw-worm flies. Structure activity relationship. J. econ. Ent., 62 (2) : 375-83.

Sharma (V. P.) et RaI (K. S.), 1967. - Sperm depletion in chemosterilized and normal males of the yellow fever mosquito Aedes aegypti. Canad. Ent., 99 (10) : 1116-18.

Stahler (N.) et Terzian (L. A.), 1963. - The response of blood fed Aedes aegypti to gamma radiation. J. econ. Ent., 56 : 416-17.

Steiner (L. F.), Mitchell (W. C.) et Baumhover (A. H.), 1962. - Progress of Fruit-Fly control by irradiation sterilization in Hawaii and the Marianas Islands. Intern. Jour. Applied Radiation Isotopes, 13 : 427-434.

Swaminathan (M. S.), Satyanirula, Natarajan (A. T.) et Sharma (R. P.), 1963. - Mutations: Incidence in Drosophila melanogaster reared on irradiated medium. Science, 141 (3581) : 637-38.

Tantawy (A. O.), Abdel Malek (A. A.) et Wakid (A. M.), 1967. - Studies on the eradication of Anopheles pharoensis by the sterile male technique using Cobalt 60 . V. Mating competitiveness in radiosterilized males. J. econ. Ent., 60 (3): 696-699.

WeidhaAs (D. E.), 1962. - Chemical sterilization of mosquitoes. Nature, Lond., 195 (4843) : 786-787.

-, Schmidt (C. H.) et Chamberlain (W. F.), 1960. - Research on radiation in insect control. Radioisotopes and Radiations in Entomology. Proc. Symp. Bombay, 5-9 déc. 1960, 257-265.

-, - et SEABRooK, 1962. - Field studies on the release of sterile males for the control of Anopheles quadrimaculatus. Mosquito News, 22 (3) : 283-91.

White (G. B.), 1966. - Chemosterilization of Aedes aegypti by pupal treatment. Nature, Lond., 210 (5) : 1372-73.

Wicht (M. C.) et HAYS (S. B.), 1967. - Effect of reserpine on reproduction of the house fly. J. econ. Ent., 60 (1) : 36-38.

YAKhimovich (L. A.) et LUSTERNAK (I. L.), 1965. - The effect of heat on the nature of the reproduction of the locust Locusta migratoria L. Dokl. Akad. Nauk. S.S.S.R., 162 : 1408-11. 